\title{
Nutritional Support: A Prophylaxis Against Stress Bleeding After Spinal Cord Injury
}

\author{
J. Kuric, RN, MSN, CCRN, C. E. Lucas, MD. A. M. Ledgerwood, MD, \\ A. Kiraly, RN, CCRN, G. G. Salciccioli, MD, C. Sugawa MD \\ Southeastern Michigan Spinal Cord Injury System, Department of Surgery and \\ Orthopedic Surgery, Wayne State University and Detroit Receiving Hospital, \\ Detroit, Michigan 48201, USA.
}

\section{Summary}

The incidence of upper gastrointestinal (UGI) bleeding and the effect of nutritional support was studied retrospectively in 166 spinal cord injured patients. Sixty six patients included in group 1 were started on oral diet when 'clinically ready' which resulted in a haphazard manner for provision of nutrition. One hundred patients in group 2 were treated according to an organised nutrition protocol. The protocol initiates total parental nutrition $(T P N)$ if the patient is not tolerating an oral diet by day 5. All group 2 patients met their total energy requirements (TER) within 48 hours after initiating caloric supplementation.

The overall incidence of acute acid peptic ulceration leading to significant bleeding or perforation was $4 \%$. Five of the 66 group 1 patients $(7.5 \%)$ and 2 of the 100 group 2 patients ( $2 \%$ ) developed acute ulcerations. While the exact mechanism remains unclear, this significant $(p<0.05)$ reduction indicates that a nutritional regimen that meets a patients TER decreases the likelihood of acid peptic complications after spinal cord injury.

Key words: Spinal cord injury; Peptic ulcer; Gastric bleeding; Nutrition.

Care of the patient with acute spinal cord injury is hampered by multiple organ system dysfunction. Attention to minute details of prophylaxis against organ insult plus aggressive intervention for established organ compromise form the basis for a successful outcome.

Gastrointestinal system dysfunction is a common sequela to acute spinal cord injury. Adynamic ileus, acute stress ulceration of the stomach and duodenum, and malnutrition reflect this impairment. This symptom complex may result, in part, from unopposed vagal stimulation which leads to gastric acid hypersecretion, gastric dilatation, relaxation of gastric sphincters, and decreased intestinal peristalsis.

A prophylactic regimen against acute stress ulceration has been operative on the acute Spinal Cord Injury Center (SCIC) at Detroit Receiving Hospital 
since it opened in January 1982; this regimen includes nasogastric (NG) suction to remove ongoing acid secretion. Consequently, enteral nutrition is delayed thereby mandating early use of the parenteral (TPN) route. During the initial 15 months after opening the acute SCIC, the regimen for nutritional support was haphazard with no prescribed protocol. This often led to inattentive delays in full nutritional support. After March 1983, a nutritional protocol was instituted for all SCIC patients.

Subsequently, a decrease in the incidence of serious gastrointestinal (GI) haemorrhage or perforation has occurred. This report highlights the effects of this nutritional protocol on the incidence of serious acid peptic disease in SCIC patients.

\section{Clinical materials}

From January 1982 until January 1985, 166 patients treated on the acute SCIC were included in this analysis. Patients with any previous history of upper GI tract pathology or associated injuries to the gastrointestinal tract were excluded. The records of these patients were analysed for age, sex, type of injury, number of associated injuries, injury severity score, level of spinal cord function, incidence of complete spinal cord injury, need for ventilatory support, nutritional support, nutritional parameters, complications related to acute stress ulceration, and outcome.

All patients were treated in a similar manner by the same multidisciplinary team. There were no significant differences between the two groups in steroid regime or surgical vs. non-surgical management. The regimen for prophylaxis against acute stress ulceration was the same throughout this time interval. This consisted of $\mathrm{H} 2$ receptor blockade by cimetidine, nasogastric (NG) suction to remove ongoing acid secretion, and hourly gastric $\mathrm{pH}$ monitoring followed by gastric acid neutralisation whenever the gastric $\mathrm{pH}$ was less than $5 \cdot 0$. This $\mathrm{pH}$ level was chosen to stay above the upper range of pepsin activity (around 4.5) and to avoid unnecessary stimulation of gastrin release due to excessive alkalinisation of the antrum.

During the initial 15 months of the spinal centre operation, caloric replacement was provided primarily by way of the gut when the patient was deemed 'clinically ready' for oral feedings or NG feedings. TPN was used only when several attempts at enteral feedings failed. Since the concept 'clinically ready' conveys a wide range of unprecise findings by multiple observers, many patients, in retrospect, were probably fed too soon resulting in aggravated gastric dilatation, worse adynamic ileus, absence of neutralisation of unabated gastric acid secretion and inappropriate delay in appropriate caloric replacement by TPN. Subsequent to March 1983, a nutritional protocol was followed. When a patient was not ready or failed to tolerate oral diet by hospital day 4 , a central line was inserted and a $10^{\circ}$, glucose solution of approximately 3 litres per day was initiated. TPN was started on day $5 ; 25^{\circ}$ o glucose and $4 \cdot 25^{\circ}{ }_{0}$ amino acid solution was started at $50 \mathrm{ml} / \mathrm{h}$ and advanced to $125 \mathrm{ml} / \mathrm{h}$ within 24 to 48 hours dependin $;$ upon estimated total energy requirement (TER) and glucose tolerance. Intralipid $\left(500 \mathrm{ml}, 20^{\circ}{ }_{0}\right)$ was added 3 times per week. Minor deviations from this regimen to adjust for associated liver, kidney, or cardiac compromise 
were made when appropriate. When oral feedings or NG tube feeding could not be implemented by day 17 or the patient required an operation for other reasons and appeared unable to tolerate oral feedings in the immediate future, a feeding jejunostomy was placed. The jejunostomy feedings were advanced as tolerated until the patient reached TER. The TPN was weaned as the jejunostomy feedings increased being sure to maintain the total caloric intake above calculated TER. Fortuitously, none of the patients sustained inadvertant pneumothorax from placement of multiple central lines via a percutaneous subclavian vein puncture, and none of the TPN catheters led to a positive culture when removed or routinely changed every 7 to 10 days. When a patient had fever of undetermined etiology, the central vein catheters were changed every 3 days, and the tips were sent for culture and sensitivity.

The caloric needs of each patient were calculated from the Harrison-Benedict Energy Equation (BEE) formula. BEE for males equals $66+(13.7 \times \mathrm{W})+$ $(5 \times \mathrm{H})-(6.8 \times \mathrm{A})$; BEE for females equals $655+(9.6 \times \mathrm{W})+(1.7 \times$ $\mathrm{H})-(4.7 \times \mathrm{A})$ where $\mathrm{W}=$ weight in kilograms, $\mathrm{H}=$ height in centimetres, and $\mathrm{A}=$ age in years. The TER equals the BEE $\times$ activity factor $\times$ a stress factor which, for skeletal trauma, equals $1 \cdot 35$. All patients treated under the guidelines of the nutritional protocol since March 1983 met their TER within 48 hours of initiating caloric supplement. The patients treated prior to March 1983 (group 1) were statistically compared to those patients treated after March 1983 (group 2) by unpaired student 't-test' or Chi Square analysis as appropriate.

\section{Results}

There were 150 males and 16 females with an average of 35.8 years. The average injury severity score was 29.74 and was similar for both groups (Table I). The vertebral injury, and the presence of a neurological deficit were also similar for both groups. There were 72 patients with vertebral fracture without neurological deficit, 34 patients with incomplete spinal cord injury, 26 patients with complete spinal cord injury with paraplegia, and 34 patients with complete spinal cord injury and quadriplegia. The level and severity of both the vertebral fracture, and the spinal cord injury were similar for both groups (Table I). $32 \%$ of group 1 patients and $28^{\circ}$ o of group 2 patients required ventilatory support for associated acute respiratory insufficiency (Table I).

The overall incidence of acute acid peptic ulceration leading either to significant bleeding or perforation was $4_{\circ}^{\mathrm{o}}$. Five of the 66 group 1 patients developed

Table I Severity of insult

\begin{tabular}{lcc}
\hline & Group 1 & Group 2 \\
\hline Number of Patients & 66 & 100 \\
Age & $37 \cdot 0$ & $34 \cdot 6$ \\
Injury severity score & $35 \cdot 9+11 \cdot 22$ & $27 \cdot 28+6 \cdot 90$ \\
No neuro deficit & $31\left(47^{\circ}{ }_{0}\right)$ & $41\left(41^{\circ}{ }_{0}\right)$ \\
Paraplegia-complete & $13\left(20^{\circ}{ }_{0}\right)$ & $13\left(13^{\circ}{ }_{0}\right)$ \\
Quadriplegia-complete & $15\left(22^{\circ}{ }_{0}\right)$ & $19\left(19^{\circ}{ }_{0}\right)$ \\
Required ventilitory support & $21\left(32^{\circ}{ }_{0}\right)$ & $28\left(28^{\circ}{ }_{0}\right)$ \\
Paraplegia-incomplete & $5\left(7 \cdot 5^{\circ}{ }_{0}\right)$ & $19\left(19^{\circ}{ }_{0}\right)$ \\
Quadriplegia-incomplete & $2\left(3^{\circ}{ }_{0}\right)$ & $9\left(8^{\circ}{ }_{0}\right)$ \\
\hline
\end{tabular}


Table II Acid - peptic complications

\begin{tabular}{lcc}
\hline & Group 1 & Group 2 \\
\hline Severe bleeding and perforation & 2 & 1 \\
Severe bleeding & 2 & 0 \\
Perforation & 1 & 1 \\
Total & $5{ }^{\star}$ & - \\
${ }^{\star} \mathrm{p}<0.05$ by Chi square analysis without Yates correction. &
\end{tabular}

severe acute ulceration compared to 2 of the 100 group 2 patients. When the patients were analysed according to the level of acute spinal cord injury, all 7 patients with major acid peptic complications had cervical injury with major neurological deficit. Five of the 66 group 1 patients and 2 of the 100 group 2 patients with cervical injury and neurologic deficit developed major ulcer complications (Table II). Three of the group 1 patients with cervical injury had perforated gastric ulcers which in 2 patients bled prior to perforation and required multiple transfusions; all 3 patients underwent subtotal gastrectomy with vagatomy for ulcers which were located along the anterior gastric wall on the lesser curvature close to the oesophagogastric junction. The remaining 2 group 1 patients with peptic ulceration had severe bleeding which required multiple transfusions and finally responded to endoscopic injection of absolute alcohol. The mean time from injury to severe bleeding or perforation in these 5 group 1 patients averaged 14 days. Both of the group 2 patients with major acid peptic complications had perforated the gastric ulcers which, in 1 patient, followed a severe haemorrhage. The ulcers were located in the same site and both patients were treated by subtotal gastrectomy and vagotomy. The mean time from injury to bleeding or perforation in these 2 patients was 11 days.

The nutritional support regimen appeared to influence the incidence of acid peptic complications in these patients with cervical injury. Because of the recurrent problems with gastrointestinal tract function, the group 1 patients did not reach their TER until an average of 15 days. In contrast, the TER was reached in an average of 2 days in the group 2 patients. Furthermore, group 1 patients required prolonged nasogastric suction averaging 13.4 days compared to 3.4 days in the group 2 patients (Table III).

Table III Nutrition and gastric response

\begin{tabular}{lcc}
\hline & Group 1 & Group 2 \\
\hline Days until enternal feeding & $15 \cdot 5 \pm 11 \cdot 48$ & $4 \cdot 6 \pm 5 \cdot 6$ \\
Days on cimetidine & $14 \cdot 0 \pm 8 \cdot 83$ & $11 \cdot 0 \pm 10 \cdot 00$ \\
Days needing antacid therapy & $16 \cdot 0^{\star} \pm 10 \cdot 63$ & $6 \cdot 0 \pm 3 \cdot 96$ \\
Days requiring nasogastric tube & $13 \cdot 4^{\star} \pm 8 \cdot 60$ & $3 \cdot 3 \pm 4 \cdot 00$ \\
\hline
\end{tabular}

${ }^{\star} \mathrm{p}<0.05$ by unpaired (two-tailed) student $t$ test.

\section{Discussion}

The incidence of acid peptic complications following acute spinal cord injury is high. Prior studies report an incidence of 4 to $22^{\circ}$, and this incidence varies with the severity and location of the acute spinal cord injury. The present 
observation of a $4 \%$ incidence of severe acid peptic complications in this acute spinal cord injury centre is highly favourable when compared to other centres (El Masri et al., 1982; Epstein et al., 1981; Globus and Ralston, 1951; Gore et al., 1981; Kewairamani, 1979; Kiwerski, 1986; Miller et al., 1975; Moolten, 1942; Soderstrom and Ducker, 1985; Walters and Silver, 1986). This excellent result may reflect the combined approach to prophylaxis against acid peptic complications by the use of a nasogastric tube to remove acid, hourly neutralisation of the gastric juice to a $\mathrm{pH}$ above 5.0, and parietal cell mass blockade with cimetidine. Although several prospective studies have compared the relative benefit of $\mathrm{H} 2$ receptor blockade versus neutralisation as a prophylaxis against acute acid peptic complications in high risk patients, the present regimen of combined therapy probably will produce the best reduction in these complications in patients with acute spinal cord injury. Clearly, this regimen of prophylaxis was successful in completely eliminating severe acid peptic complications in all patients with thoracic or lumbar cervical spinal cord injuries.

The increased morbidity and mortality associated with acute cervical spinal cord injury has been observed by others. This level of injury creates more extensive interference with multiple organ function and creates a much greater challenge to prevent complications of the cardiovascular system, the pulmonary system, the gastrointestinal system, and the genital urinary system. The combined regimen of acid peptic prophylaxis in these very high risk patients with complete cervical spinal cord injury is inadequate since $7 \cdot 5^{\circ}$ of the group 1 patients with acute complete cervical spinal cord injury developed major acid peptic complications. Apparently, the addition of an early prophylactic nutritional support regimen augments the combined stress ulcer prophylaxis provided by the neutralisation of acid secretion and the parietal cell mass blockade. The incidence of major acid peptic complications in the 100 group 2 patients with acute complete cervical spinal cord injury was $2^{\circ}{ }_{o}$ which is less than that reported in other series (El Masri et al., 1982; Epstein et al., 1981; Kawairamani, 1979; Kiwerski, 1986; Sonderstrom and Ducker, 1985; Walters and Silver, 1986) when comparably injured patients are compared. The mechanism whereby early and effective nutritional support reduces the incidence of major acid peptic morbidity is unclear. Possibly the TPN decreases the amount of acid secretion from the parietal cell mass. The mechanism for such an inhibition is unclear, but may possibly be related to amino acid metabolites which modify the parietal cell response to both neural and humeral stimulation. Such mediators may be part of the TPN supplementation in this setting.

Alternatively, the apparent benefit of this nutritional support regimen may be indirect. For example, the early use of total parenteral nutrition eliminates the urgency to initiate enteral feedings by oral or nasogastric tube feedings. By eliminating this need or delaying the use of the compromised gastrointestinal tract until one is absolutely certain of restoration of gastrointestinal tract function, the group 2 patients may have had less problems with adynamic ileus, unneutralised acid secretion due to premature removal of the nasogastric tube, and less pulmonary dysfunction due to the effects of adynamic ileus on the diaphragm or the effects of gastric distension, vomiting, and aspiration. Regardless of the underlying mechanism, the end result identifies the benefit of an early nutrition replacement regimen, not only in terms of providing better caloric 
replacement, but also in preventing problems related to acid peptic ulceration in these high risk patients.

\section{References}

El Masri W, Cochrane P, Silver JR 1982 Gastrointestinal bleeding in patients with acute spinal injuries. Injury 14:161-167.

EPSTEIN N, Hood DC, RANSOHOFF J 1981 Gastrointestinal bleeding in patients with spinal cord trauma. Fournal of Neurosurgery 54:16.

GlobUS JH, RALSTON BL 1951 Multiple erosions and acute perforations of the esophagus, stomach, and duodenum in relation to disorders of nervous system.' fournal Mt Sinai Hospital 17:817.

GoRe RM, Mintzer RA, CALENOFF L 1981 Gastrointestinal complications of spinal cord injury. Spine 6:538-544.

KEWALRAMANI LS 1979 Neurogenic gastroduodenal ulceration and bleeding associated with spinal cord injuries. Trauma 19:259-265.

KIWERSKI J. 1986 Bleeding from the alimentary canal during the management of spinal cord injury patients. Paraplegia 24:92-96.

Miller LS, Stass WE, Herbison GJ 1975 Abdominal problems in patients with spinal cord lesions. Archives of Physical Medicine and Rehabilitation 56:405-408.

MOOLTEN SE 1942 Duodenal ulcer following acute injury of the spinal cord. Fournal Mt Sinai Hospital 8:868.

SODERSTROM CA, DUCKer TB 1985 Increased susceptibility of patients with cervical cord lesions to peptic gastrointestinal complications. The fournal of Trauma 25:1030-1036.

WALTERS K, SILVER SR 1986 Gastrointestinal bleeding in patients with acute spinal injuries. International Rehabilitation Medicine 8:44-48. 